\title{
Offshore Oilfield Hybrid Renewable Power Systems Based on Al Algorithm
}

\author{
Qingguang $\mathrm{Yu}^{1,}{ }^{*}$, Zhicheng Jiang ${ }^{1}$, Yuming Liu ${ }^{1}$ and Gaoxiang Long ${ }^{1}$ \\ ${ }^{1}$ State Key Laboratory of Power Systems, Department of Electrical Engineering, Tsinghua University, 100084 Beijing, China
}

\begin{abstract}
This paper proposes a structural optimization model for the offshore oilfield interconnected power system. The model focuses on evaluating the reliability of the system. It is found that the $\mathrm{N}-1$ fault is the primary fault mode leading to severe power loss due to the probability of fault occurrence and the fault consequence according to the statistics of the historical fault information of the offshore oilfield power system. Considering the characteristics of the offshore oil extraction process, the priority of load removal in different processes under different fault conditions is different. Comprehensively considering the above factors, the model uses the minimum load shedding model that considers the load priority level in the objective function to calculate the power outage losses in all $\mathrm{N}-1$ fault states of the system. The test results of numerical examples prove that the optimized solution of the structural optimization model can achieve a better balance between economy and reliability.
\end{abstract}

\section{Introduction}

With the expansion of the scale of offshore oil extraction, the offshore oilfield power system has begun to shift towards multi-platform interconnected power systems. The generator configuration scheme and platform connection scheme become the key factors to improve reliability at a certain economic level. Firstly, this paper proposes a structural optimization model for the offshore oilfield interconnected power system. The model focuses on evaluating the reliability of the system. It is found that the $\mathrm{N}-1$ fault is the primary fault mode leading to severe power loss due to the probability of fault occurrence and the fault consequence according to the statistics of the historical fault information of the offshore oilfield power system. Considering the characteristics of the offshore oil extraction process, the priority of load removal in different processes under different fault conditions is different. Comprehensively considering the above factors, the model uses the minimum load shedding model that considers the load priority level in the objective function to calculate the power outage losses in all $\mathrm{N}-1$ fault states of the system. The test results of numerical examples prove that the optimized solution of the structural optimization model can achieve a better balance between economy and reliability.

\section{Offshore oilfield hybrid renewable power systems}

\subsection{Overview of offshore oilfield power system research}

Offshore oil exploitation requires very high reliability of power system. The main driving force for the development of offshore oil field power system is to continuously pursue higher system reliability under the premise of acceptable economy so as to ensure offshore oil exploitation. At present, there are two research trends in improving the reliability of offshore oilfield power system. The starting point of both schemes is to improve the power supply capacity of offshore oilfield power system, improve the shock resistance capability of the power system, and thus guarantee the operation reliability of the system.

The first solution is to introduce shore power into the offshore oil field power system by using the flexible DC transmission technology. By transferring shore power to the offshore platform through the flexible DC transmission technology, the offshore oil field power system can have infinite power supply just like the onshore power system, thus greatly improving the reliability of the power generation system. At present, many studies[1] have verified the feasibility of introducing shore power into the power system of offshore oil fields by using the flexible DC transmission technology, and put forward the analysis and design scheme of the offshore oil field flexible DC power transmission system completely. But if offshore oil from land far away, the submarine cable construction costs are high, and if the connection is on land and offshore platform with one loop submarine cable, so the submarine cable would be on the weak links of

* Corresponding author: yuqingguang@163.com 
transmission system, once the submarine cable fails, the whole face blackouts in offshore oil field production, rely on single submarine cable connection on land and offshore platforms is very difficult to guarantee the reliability of offshore platform, laying of submarine cable or two in offshore platform configuration generator will lead to project cost increased; In addition, the introduction of shore power by using flexible direct current transmission technology requires the construction of bulky converter stations on offshore platforms, which also greatly increases the construction cost of offshore platforms. In a word, the scheme of introducing shore power by using flexible DC technology is not economically good[2] and cannot achieve the balance between economy and reliability, so it has not been widely applied at present.

The second solution is more traditional interconnected power system in offshore oil field, the starting point of the solution is to increase the same power supply capacity, make each power station platform can support mutually share the spare capacity, make the power generation system both in economy and reliability are improved, and relative to the introduction of shore power scheme, the scheme of new submarine cable length is short, don't need to do massive changes to the existing platform, stronger economy, at present, a lot of offshore platforms interconnected power system has started a number of small-scale construction. Literature gives a detailed introduction of the current construction status of offshore multi-platform interconnected power system. Literature studies the safe and stable operation of the existing offshore multi-platform interconnected power system. Currently offshore platform more interconnected power system is the land grid technology application directly to the offshore oil field, due to space limitations and the high costs of submarine cable investment platform, many land power grid to improve the reliability of the new technologies have not been applied in electric power system in offshore oil field, and even many mature technologies due to the technical innovation is difficult also failed to has been applied in offshore oil field, offshore platform more interconnected power system, therefore, is a simplified version of land power system, the reliability level is far lower than the land power system.

Due to the power system in offshore oil field is at a monophyletic radiation to turn to the early stages of the interconnected system of offshore platform more interconnected power system network scale is generally small, the project networking options is less, the need for structural optimization is not high, so on offshore platform more interconnected power system structure optimization literature are rare. Only literature [3] is devoted to the structural optimization of offshore multiplatform interconnected power systems. In this paper, a power-grid collaborative optimization model is established. The objective function of this model takes into account the generator construction cost, sea cable construction cost, system operation cost and power failure loss cost. The decision variables include wire corridor, generator platform and generator number. But the literature is still belong to direct the land power system planning technology directly applied to offshore platform more interconnected power system, the method has the defects of the following several aspects: first, to improve the efficiency of calculation, the optional submarine cable type simplified, but the actual situation, different specifications of the submarine cable had a great influence on the overall cost of the construction plan; Secondly, in the calculation of power failure cost, the calculation of power shortage is the difference between the total installed capacity and the total load, without considering the loss of power failure caused by the limitation of network capacity in the case of failure. Finally, the closed-loop design is only applied to the optimization system, without fully considering the operating conditions of the open-loop system.

\subsection{Overview of power system planning research}

The contents of power system planning include power supply, network frame, tie line, reactive compensation equipment, distributed power supply, etc. According to these planning contents, power system planning can be divided into power supply planning[4-5], power grid planning[6-7], tie line planning[8], reactive power planning, and distributed power supply planning[9]. These plans can be divided into three stages according to the power system design process, usually the design of the latter stage needs to be based on the design results of the previous stage. The first step is power planning, which usually divides the power supply area based on the minimum load moment and determines the location and size of the power supply in combination with the actual geographical conditions. The second link is power network planning. The main research content is to plan the connection relationship between power supply and load with economic efficiency and reliability as long as the power supply planning is completed. The third part is the rest of the tie lines planning, reactive power planning and distributed power planning, the planning is done in the power supply and network frame design, based on the research content is to optimize the tie lines planning system of tie line number and link of the erect position, link main grid is redundant power supply circuit, the existence of the tie line can guarantee in some main line fault broken case load can still get normal power supply, link to improve the system reliability has important significance, Reactive power planning research content is in the optimal allocation of reactive power compensation equipment in each node configuration of each node voltage to run in the normal range at the same time minimizing the total cost of reactive power compensation equipment configuration, distributed power planning research content is optimizing the allocation of each node of the distributed power supply capacity in order to improve the reliability of the system or operational economy. However, with the improvement of computer computing power, more and more studies[10] began to integrate multiple planning contents into one planning work for collaborative planning, so that better planning results could be 
obtained compared with individual planning. The structural optimization of offshore multi-platform interconnected power system studied in this paper is to combine the power supply planning, power grid planning and tie line planning, and to achieve a more balanced scheme of economy and reliability through the coordinated optimization of power supply - network frame - tie line.

Power system planning can be divided into singlestage planning[12] and multi-stage planning[11] according to whether load changes with time are considered. In particular, single-stage planning considers that load is unchanged in the planning period and only considers the new load within the planning period without considering the new load outside the planning period. Multi-stage planning subdivides the planning period into several stages, and each stage corresponds to a different load level. Based on the load level of each stage, the unit configuration scheme and network frame connection scheme of each stage are planned. Compared with single stage planning, multi-stage planning can achieve global optimal in a longer time scale by optimizing and synthesizing the transition of each stage. The global optimal degree that multi-stage programming can achieve is positively correlated with the level of subdividing the planning time period. However, the more detailed the planning time period is divided, the more decision variables will increase and the programming model will become more complex, which brings great trouble to model solving. In this paper, considering the offshore oil platform production there is a special electric power does not decrease output, a small change in the planning period load levels, at the same time because of the unpredictability of offshore drilling are difficult to predict reservoir position location and an oil platform construction under its load, so offshore oil platform structure of interconnected power system optimization planning model suitable for single phase.

According to the optimization objectives, the power system planning can be divided into economic model, reliability model and comprehensive model ${ }^{[13]}$. Economic model is the most widely used model at present. This model only considers the investment cost of generator and network frame and system operation cost. In terms of reliability, the system is required to meet normal operation or pass $\mathrm{N}-1$ verification. The reliability model takes the reliability index as the optimization goal, and the optimized scheme also needs the final screening in terms of economy. Literature [13] selects the network destruction resistance measurement index to optimize the alternative network structure with high availability. At present, the reliability model which only takes the reliability as the optimization goal is rarely applied. Comprehensive model is considered in the optimization model of the economy, reliability and comprehensive model considering the different indicators mainly has two ways: one is the different indicators through weighted aggregation, the difficulties in the treatment of multiple objective is to need to reasonably determine the weights of different indexes, for larger orders of magnitude difference index also need to do the normalized processing; The other is a multi- objective optimization model is set up, the multiobjective model looks good, but the multi-objective optimization model for pareto optimality usually stop optimization criterion, in the solution of multi-objective optimization space that conform to pareto optimal solutions of many a day, and multi-objective optimization model is therefore difficult to get a really achieve a balance between multi-objective scheme. Offshore platform more interconnected power system structure optimization using a comprehensive model considering economy and reliability are two important optimization objectives, including through the use of the reliability index representing fault outage loss cost, makes the economy between target and reliability can be simple sum, avoids the trouble determining the weight.

Optimization model for power system planning is a MINLP problem, heuristic algorithm can directly only, heuristic algorithm including genetic algorithm, particle swarm optimization (PSO), simulated annealing algorithm, ant colony algorithm, tabu search, the algorithm to the optimization model of requirement is small, is not sensitive to optimization model of the scale, but these algorithms can guarantee the global optimal solution is obtained, the algorithm easy to fall into local optimum, the current of heuristic algorithms are improved by adjusting the parameters within an acceptable time to avoid algorithm into local optimum in order to get a good solution acceptable engineering sense. In order to solve the problem by using accurate mathematical programming methods, many studies have simplified the mixed integer nonlinear model to reduce the order of the higher-order constraints in the optimization model, such as piecewise linearization, using the $\mathrm{DC}$ power flow equation or the $\mathrm{AC}$ power flow equation retaining the quadratic term instead of the $\mathrm{AC}$ power flow equation, etc. These simplified models are usually mixed Integer Quad constrained program (MIQCP) with quadratic terms in constraints or mixed Integer Linear Programming (MILP), so that precise solutions can be obtained using commercial solders such as CPLEX and Gurobi, However, in general, these optimization models that can reduce dimension only consider the economy of the scheme, that is, the economic model is used, and the reliability only requires that the scheme can run normally. Therefore, when studying the structural optimization of offshore multiplatform interconnected power system, the mixed integer nonlinear model is still established to ensure the reliability of the model can be fully considered. However, the model is simplified to some extent by using DC power flow algorithm instead of $\mathrm{AC}$ power flow algorithm.

\section{Al algorithm of Offshore OilFleld Power System}

\subsection{Structure of offshore oilfield platform}

The offshore oil platforms are interconnected by $35 \mathrm{kV}$ cables. Each offshore oil platform contains multiple 
transformers and loads, most of which are inductive loads. The connection mode is shown in Fig. 1. It is the location of offshore oil platform group power grid, G1G4 are central platform, G5 are wind farm and photovoltaic power station.

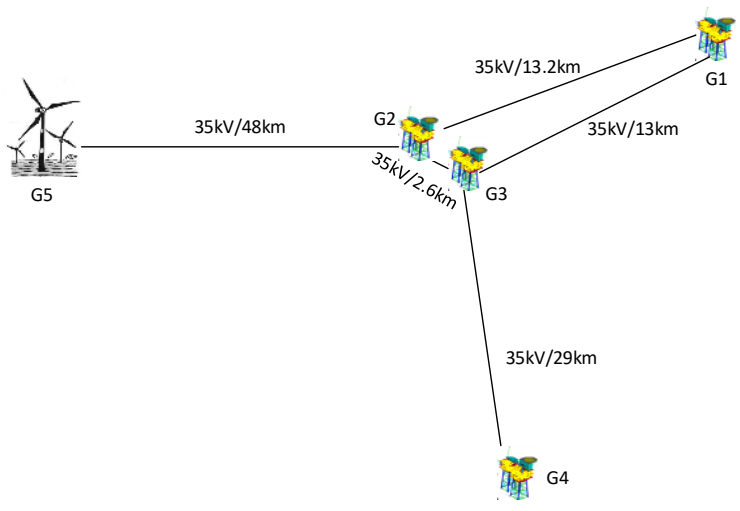

Fig. 1. Structure of offshore oil platform connection.

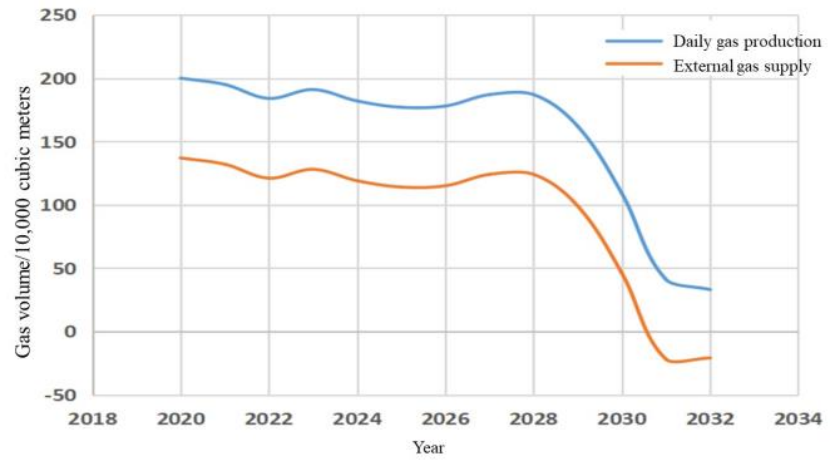

Fig 2. Daily production trend curves of associated gas in Oilfield

Offshore oil and gas fields in the process of drilling, usually with the output of oil field associated gas. Most offshore platforms, are equipped with corresponding gas turbine units. Associated gas can be used as power generation fuel to reduce the amount of offshore gas purchased and increase economic benefits, which is a reasonable way to use associated gas. For some platform of Oil and Gas Field, daily production forecast of associated gas in different years is shown in Fig. 2. It is estimated that 2 million cubic meters of associated gas will be produced daily in 2020, and 630,000 cubic meters will be generated by gas turbine units. The platform is equipped with undersea natural gas pipelines, and 1.37 million cubic meters can be transported to land terminals. With the increase of years, the output of associated gas in the oilfield shows a downward trend. By 2031, the daily production of associated gas will be 410,000 square meters. When one gas unit is used for 8 units, the associated gas is no longer enough to supply the gas volume of the unit, so natural gas needs to be purchased from outside.

\subsection{Structure of hybrid renewable power system}

As shown in Fig.3, the structure of hybrid renewable power system is composed of photovoltaic power station, wind farm power station, and battery storage power system, together with Gas turbine and Associated Gas turbine and Load.

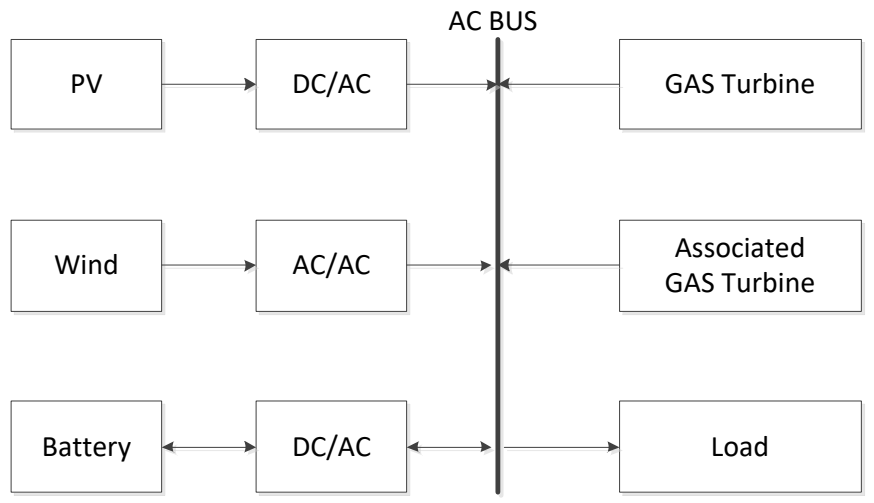

Fig. 3. Structure of hybrid renewable power system

According to the weather changes in the weather conditions, scenery complementary wind power system is mainly under four kinds of operation mode: the wind working together with photovoltaic power generation, wind working alone, photovoltaic power generation working alone, battery or Gas turbine working alone. At the same time because of the wind power, photovoltaic power, storage battery charging and discharging power and load power with time and dynamic, each operation mode can be subdivided into different working states. As the weather conditions and load conditions at any time change, working mode and working state of the system are corresponding to change. The control system requires real-time monitoring, therefore, wind turbines, photovoltaic array, battery and load conditions. For wind turbines, photovoltaic array, battery and coordinated control of Gas turbine, common coordination control is necessary.

\subsection{Al algorithm of offshore oilfield power system}

Artificial neural network (ANN) is composed of neurons connected in the great of an adaptive nonlinear dynamic system. Although the structure and function of each neuron is simpler, but a large number of neurons combination system of their behavior are very complex. ANN prediction model is generally composed of input layer, output layer and one or more hidden layers. By error back propagation method, after training can get approximation nonlinear model. There are two objectives for the power flow optimisation mentioned in the flowchart. The first is to ensure that the system outage cost is minimal. The second is to ensure that all loads are powered by their nearest generator platforms, which can reduce the power flow on the tie line between generator platforms, resulting in the selection of smaller conductors to improve the economy of the scheme. The first optimisation goal takes precedence over the second.

\section{Numerical results}


As shown in Fig. 4, which is based on certain oilfield in the south China sea waters, the group of test case, the oilfield group has established 9 platform, one platform 1 is built on the island of terminal treatment plant, the node is equipped with 7 rated capacity of $6000 \mathrm{kw}$ generators, platform and platform 62 platform for existing power stations, respectively with three rated capacity of $4261 \mathrm{kw}$ generators and two (2) the rated capacity of $2800 \mathrm{kw}$ generators, platform $3 \sim 5$, and $7 \sim$ 9 for wellhead platform has been built, platform $1 \sim 5$ and $6 \sim 9$ has respectively into two independent radial power system, expand the scale of development, now oilfield. It is expected to build a total of 11 platforms from 10 to 20 in the future, among which platform 12 is a newly built power station platform. According to the overall load condition of the system, it is estimated that generators with rated capacity of $7600 \mathrm{~kW}$ can be installed.

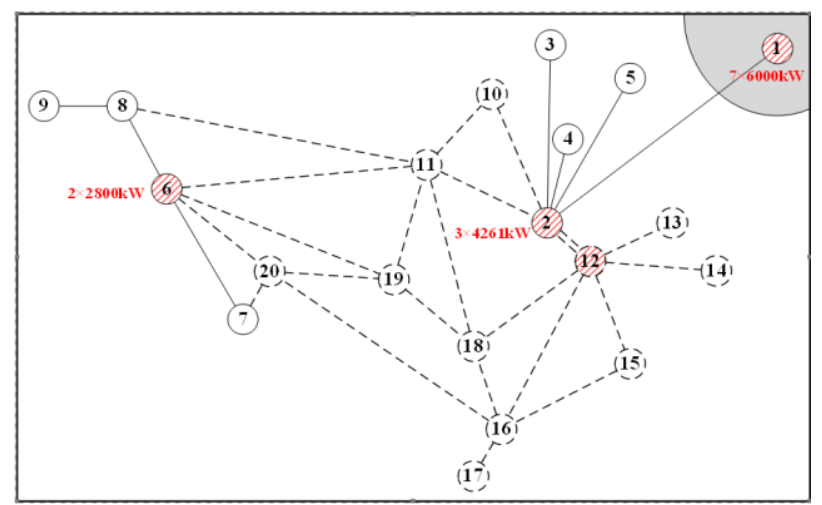

Fig. 4. Offshore oilfield power system

\section{Conclusions}

In this paper, the offshore oilfield power system research state and power system planning research state are reviewed, an offshore oilfield hybrid renewable power system prototype is carried out, and the ANN algorithm is discussed to optimize the structure. Considering the characteristics of the offshore oil extraction process, the priority of load removal in different processes under different fault conditions is different. Comprehensively considering the above factors, the model uses the minimum load shedding model that considers the load priority level in the objective function to calculate the power outage losses in all $\mathrm{N}-1$ fault states of the system. The test results of numerical examples prove that the optimized solution of the structural optimization model can achieve a better balance between economy and reliability.

\section{Acknowledgements}

This project is supported by the National Key R\&D Program of China (2018YFB0904800).

\section{References}

1. Li, X., Zhang, A., Jing, J., et al.: 'Overview of Offshore Electric Systems', Power System and Clean Energy, 2016, 32, (2), pp. 1-7.

2. Zhou, X., Lv, Y., Li, Y., et al.: 'Power Grid Interlinking Engineering Technology for Offshore Island' (Tsinghua University Press, 2013)

3. Sun D, Xie X, Wang J, et al. 'Integrated generationtransmission expansion planning for offshore oilfield power systems based on genetic Tabu hybrid algorithm'. Journal of Modern Power Systems and Clean Energy, 2017,5(1):pp 117-125.

4. Ravadanegh S N, Roshanagh R G. 'A heuristic algorithm for optimal multistage sizing, siting and timing of MV distribution substations'. Electric Power Systems Research, 2013,105:pp 134-141.

5. El-Fouly T H M, Zeineldin H H, El-Saadany E F, et al. A new optimization model for distribution substation siting, sizing, and timing. International Journal of Electrical Power \& Energy Systems, 2008,30(5):pp 308-315.

6. Bhowmik S K G P. Distribution System Planning Through Combined Heuristic and Quadratic Programing Approach. Electric Machines \& Power Systems, 2000,28(1):pp 87-103.

7. Moreira J C, Miguez E, Vilacha C, et al. LargeScale Network Layout Optimization for Radial Distribution Networks by Parallel Computing. IEEE Transactions on Power Delivery, 2011,26; 26(3; 3):pp 1946-1951.

8. Hou K, Rim J, Jia H, et al. Optimal Planning of Urban Distribution Network Tie-Line with Coordination of Reliability and Economics. Tianjin Daxue Xuebao (Ziran Kexue yu Gongcheng Jishu Ban)/Journal of Tianjin University Science and Technology, 2019,52(12):pp 1293-1302.

9. Tan Y, Wang W. Multi-objective optimization of distributed generation considering economic benefits. Hunan Daxue Xuebao/Journal of Hunan University Natural Sciences, 2015,42(10):pp 89-96.

10. G. M, J. C, J. M A. Joint Expansion Planning of Distributed Generation and Distribution Networks. IEEE Transactions on Power Systems, 2015,30(5):pp 2579-2590.

11. Tabares A, Franco J F, Lavorato M, et al. Multistage Long-Term Expansion Planning of Electrical Distribution Systems Considering Multiple Alternatives. IEEE Transactions on Power Systems, 2016,31; 31(3; 3):pp 1900-1914.

12. Jabr R A. Polyhedral Formulations and Loop Elimination Constraints for Distribution Network Expansion Planning. IEEE Transactions on Power Systems, 2013,28; 28(2; 2):pp 1888-1897.

13. Franco J F, Rider M J, Romero R. A mixed-integer quadratically-constrained programming model for the distribution system expansion planning. International Journal of Electrical Power \& Energy Systems, 2014,62:pp 265-272. 